\title{
Second-order stochastic differential equation model as an alternative for the ALT and CALT models
}

\author{
J.H.L. Oud
}

Received: 23 October 2009 / Accepted: 13 February 2010 / Published online: 26 May 2010

(C) The Author(s) 2010. This article is published with open access at Springerlink.com

\begin{abstract}
The paper first discusses the autoregressive latent trajectory (ALT) model and presents in detail its improved version, the continuous-time autoregressive latent trajectory (CALT) model. Next, serious problems related to the linear components in the ALT and CALT models are dealt with. As an alternative for the linear component, the first-order derivative in a second-order stochastic differential equation model is proposed. This is applied to Marital Satisfaction data, collected in four consecutive years (2002-2005). It is pointed out that the first-order derivative as explanatory variable has none of the problems associated with the linear component.
\end{abstract}

Keywords Continuous-time autoregressive latent trajectory model $\cdot$ Second-order stochastic differential equation model $\cdot$ Structural equation modeling $\cdot$ Exact discrete model $\cdot$ Random slopes $\cdot$ Asymptotic stability $\cdot$ Time-specific $\cdot$ Time-varying

\section{Introduction}

Curran and Bollen (2001) and Bollen and Curran (2004, 2006) introduced the autoregressive latent trajectory (ALT) model. This model combines two very popular models in behavioral science, the autoregressive (AR) cross-lagged model and latent trajectory (LT) model. The latter is also called latent growth curve model, latent curve model, or growth curve model. AR models and LT models have typically been viewed as adversarial in the analysis of longitudinal data. Bollen and Curran, however, argued that, theoretically, there are many instances when both the processes

The author thanks Manuel Völkle and two anonymous reviewers for their valuable comments to an earlier version of this paper.

J.H.L. Oud $(\bowtie)$

Behavioural Science Institute, Radboud University Nijmegen, Nijmegen, The Netherlands

e-mail: j.oud@pwo.ru.nl 
described by the AR model and the processes described by the LT model are plausible. This made them work toward a synthesis of both approaches, resulting in the autoregressive latent trajectory (ALT) model, which captures key features of both.

The discrete-time approach in the ALT model has been criticized by Delsing and Oud (2008). They proposed a continuous-time version of the ALT model, using stochastic differential equations, called by them the continuous-time autoregressive latent trajectory (CALT) model.

In the present paper, the linear component appearing in both the ALT and the CALT models will be criticized on several counts. The linear component competes with the AR component in the ALT and CALT models but does not give clear additional information. Further, it makes these models necessarily unstable as well as time-unspecific and time-varying. It is shown that the problems associated with the linear component are solved by a second-order stochastic differential equation model. This model replaces the problematic linear component by the first-order derivative as an explanatory variable. A research example in the field of marital satisfaction illustrates the application of the alternative model.

\section{Continuous-time autoregressive latent trajectory (CALT) model}

The arguments for replacing a discrete-time model by a continuous-time model are manifold. An overview is given by Oud (2007). Most behavioral science processes evolve in continuous time. Discrete-time methods are often used to describe continuous-time processes approximately, especially in case no analytic solutions exist. This may work well, as long as the time interval used in the approximation is small. However, with typical measurement frequencies of only one or two times a year in behavioral science, discrete-time modeling in terms of the measurement interval easily becomes an oversimplification and even a distortion of reality. In addition, estimating causal effects in studies based on different discrete-time intervals makes it impossible to compare the results found. The strength and order of magnitude of the effects vary with the discrete time interval chosen by the researcher; even the sign may reverse when passing from one interval to the other or from discrete to continuous time. Because, additionally, the effects found for equal observation intervals cannot be generalized to other intervals either, discrete-time modeling in terms of large observation intervals, in spite of its popularity in behavioral science, in fact turns out to be useless.

The CALT model, a continuous time version of the ALT model, takes the following form:

$$
\begin{aligned}
\frac{\mathrm{d} \mathbf{x}(t)}{\mathrm{d} t} & =\mathbf{A x}(t)+\mathbf{B u}(t)+\boldsymbol{\kappa}+\left(\boldsymbol{\mu}_{\beta}+\boldsymbol{\kappa}_{\beta}\right) t+\mathbf{G} \frac{\mathrm{d} \mathbf{W}(t)}{\mathrm{d} t}, \\
\mathbf{y}_{t_{i}} & =\mathbf{C x}\left(t_{i}\right)+\mathbf{D u}\left(t_{i}\right)+\mathbf{v}_{t_{i}} .
\end{aligned}
$$

It consists of a stochastic differential equation (1), describing the evolution of the latent variables in $\mathbf{x}(t)$ in continuous time, and a measurement equation (2), describing for all observed variables in $\mathbf{y}_{t_{i}}$, how the latent variables in continuous time become manifest at the discrete observation time points $t_{i}$. 
The elements of $\mathbf{W}(t)$ contain the Wiener process (see, e.g., Arnold 1974; Kuo 2006). In addition to the Wiener process, which by definition is normally distributed, also the initial state variables in $\mathbf{x}\left(t_{0}\right)$ are assumed to be normally distributed, $\mathbf{x}\left(t_{0}\right) \sim$ $N\left(\boldsymbol{\mu}_{x_{t_{0}}}, \boldsymbol{\Phi}_{x_{t_{0}}}\right)$, as well as the measurement errors, $\mathbf{v}_{t_{i}} \sim N\left(\mathbf{0}, \mathbf{R}_{t_{i}}\right)$. Drift matrix $\mathbf{A}$ (auto-effects on the diagonal and cross-effects off-diagonally) is analogous to the autoregression matrix (autoregressions on the diagonal and cross-lagged effects offdiagonally) in discrete time. In fact, the autoregression matrix will be derived as a nonlinear function of the drift matrix and observation interval. Important properties of the model depend on the drift matrix.

Multiplied by matrix $\mathbf{G}$, the standard multivariate Wiener process $\mathbf{W}(t)$, having covariance matrix $\mathbf{I}$ at $t=1$, is transformed into a more general Wiener process with covariance matrix $\mathbf{Q}=\mathbf{G G}^{\prime}$ at $t=1$ (Ruymgaart and Soong 1985, pp. 68-75), called diffusion matrix. Analogously to the relation between discrete-time autoregression matrix and drift matrix, the discrete-time error covariance matrix is derived as a nonlinear function of the diffusion matrix, drift matrix, and observation interval.

Effects $\mathbf{B u}(t) \neq \mathbf{0}$ and $\mathbf{D u}\left(t_{i}\right) \neq \mathbf{0}$ for fixed exogenous variables in $\mathbf{u}(t)$ accommodate for nonzero and nonconstant mean trajectories $E[\mathbf{x}(t)]$ and $E\left(\mathbf{y}_{t_{i}}\right)$ that are frequently observed even in the case of an asymptotically stable model.

By means of vector $\boldsymbol{\kappa}$, state equation (2) introduces constant (over time) random subject effects $\boldsymbol{\kappa} \sim N\left(\mathbf{0}, \boldsymbol{\Phi}_{\kappa}\right)$, which are referred to as intercept variables. They enable to distinguish intercept variance (unobserved heterogeneity between subjects) clearly from stability, avoiding the artifact of using instability (high autoregression) to keep the subject trajectories apart. Note that the covariance matrix $\boldsymbol{\Phi}_{\kappa, x_{t_{0}}}$ between initial state and intercept cannot, in general, be assumed zero, because the intercept variables $\boldsymbol{\kappa}$ are modeled to influence $\mathbf{x}(t)$ continuously, after but also already before $t_{0}$. Analogously, the slope variables $\boldsymbol{\mu}_{\beta}+\boldsymbol{\kappa}_{\beta}$ with means $\boldsymbol{\mu}_{\beta}$ and covariance matrices $\boldsymbol{\Phi}_{\kappa_{\beta}}, \boldsymbol{\Phi}_{\kappa_{\beta}, x_{t_{0}}}$, and $\boldsymbol{\Phi}_{\kappa_{\beta}, \kappa}$ are introduced.

It should be noted that random subject effects can be added to state vector $\mathbf{x}(t)$, so making the continuous-time CALT model and analogously the discrete-time ALT model and its submodels (AR and LT) accessible for basic state-space model estimation procedures. Intercepts $\boldsymbol{\kappa}$ and slope effects $\left(\boldsymbol{\mu}_{\beta}+\boldsymbol{\kappa}_{\beta}\right) t$ would get $\mathbf{0}$ and $\boldsymbol{\mu}_{\beta} t_{0}$ in $E\left[\mathbf{x}\left(t_{0}\right)\right]$, and both $\mathbf{0}$ in $\mathbf{C}$ (unobserved heterogeneity). However, whereas $\boldsymbol{\kappa}$, being constant over time, gets also $\mathbf{0}$ in $\mathbf{A}$ because of $\frac{\mathrm{d} \kappa}{\mathrm{d} t}=\mathbf{0}$, the situation with regard to $\boldsymbol{\kappa}(t) \equiv\left(\boldsymbol{\mu}_{\beta}+\boldsymbol{\kappa}_{\beta}\right) t$ in the dynamic state equation is slightly more complicated: $\frac{\mathrm{d} \kappa(t)}{\mathrm{d} t}=\frac{1}{t} \boldsymbol{\kappa}(t)=\boldsymbol{\mu}_{\beta}+\boldsymbol{\kappa}_{\beta}$. Nevertheless, CALT model (1)-(2) turns out to be a special case of the more basic continuous-time state space model (3)-(4):

$$
\begin{aligned}
\frac{\mathrm{d} \mathbf{x}(t)}{\mathrm{d} t} & =\mathbf{A x}(t)+\mathbf{B u}(t)+\mathbf{G} \frac{\mathrm{d} \mathbf{W}(t)}{\mathrm{d} t}, \\
\mathbf{y}_{t_{i}} & =\mathbf{C x}\left(t_{i}\right)+\mathbf{D u}\left(t_{i}\right)+\mathbf{v}_{t_{i}} .
\end{aligned}
$$

For estimating the CALT model in form (1)-(2) or general state space form (3)-(4) in the case of a sample of multiple subjects, we apply the EDM/SEM procedure (Oud and Jansen 2000). The EDM, introduced in 1961-1962 by Bergstrom (1988), links in an exact way the discrete-time model parameters to the underlying continuous-time 
model parameters by means of nonlinear constraints. The link is made by solving the stochastic differential equation as given for the CALT model in (1) for the correct observation intervals $\Delta t_{i}=t_{i}-t_{i-1}$ and thus relating the discrete-time parameters in the discrete-time equation

$$
\begin{gathered}
\mathbf{x}_{t_{i}}=\mathbf{A}_{\Delta t_{i}} \mathbf{x}_{t_{i}-\Delta_{t_{i}}}+\mathbf{B}_{\Delta t_{i}} \mathbf{u}_{t_{i}-\Delta_{t_{i}}}+\mathbf{H}_{\Delta t_{i}} \boldsymbol{\kappa}+\mathbf{F}_{\Delta t_{i}} \boldsymbol{\mu}_{\beta}+\mathbf{F}_{\Delta t_{i}} \boldsymbol{\kappa}_{\beta}+\mathbf{w}_{t_{i}-\Delta_{t_{i}}} \\
\text { with } \operatorname{cov}\left(\mathbf{w}_{t_{i}-\Delta_{t_{i}}}\right)=\mathbf{Q}_{\Delta t_{i}}
\end{gathered}
$$

to the underlying ones in (1) by the highly nonlinear restrictions on the discrete-time parameters, given in (6):

$$
\begin{aligned}
\mathbf{A}_{\Delta t_{i}} & =\mathrm{e}^{\mathbf{A} \Delta t_{i}}, \\
\mathbf{B}_{\Delta t_{i}} & =\mathbf{A}^{-1}\left(\mathbf{A}_{\Delta t_{i}}-\mathbf{I}\right) \mathbf{B} \\
\mathbf{H}_{\Delta t_{i}} & =\mathbf{A}^{-1}\left(\mathbf{A}_{\Delta t_{i}}-\mathbf{I}\right) \\
\boldsymbol{\Phi}_{\kappa_{\Delta t_{i}}} & =\mathbf{H}_{\Delta t_{i}} \boldsymbol{\Phi}_{\kappa} \mathbf{H}_{\Delta t_{i}}^{\prime}, \\
\boldsymbol{\Phi}_{\kappa_{\Delta t_{i}}, x_{t_{0}}} & =\mathbf{H}_{\Delta t_{i}} \boldsymbol{\Phi}_{\kappa, x_{t_{0}}} \text { for } \boldsymbol{\kappa}_{\Delta t_{i}}=\mathbf{H}_{\Delta t_{i}} \boldsymbol{\kappa}, \\
\mathbf{F}_{\Delta t_{i}} & =\mathbf{H}_{\Delta t_{i}} t_{i-1}+\mathbf{A}^{-1}\left(\mathbf{H}_{\Delta t_{i}}-\Delta t_{i} \mathbf{I}\right) \\
\boldsymbol{\Phi}_{\kappa_{\beta, \Delta t_{i}}} & =\mathbf{F}_{\Delta t_{i}} \boldsymbol{\Phi}_{\kappa_{\beta}} \mathbf{F}_{\Delta t_{i}}^{\prime}, \\
\boldsymbol{\Phi}_{\kappa_{\beta, \Delta t_{i}}, \kappa_{\Delta t_{i}}} & =\mathbf{F}_{\Delta t_{i}} \boldsymbol{\Phi}_{\kappa_{\beta}, \kappa} \mathbf{H}_{\Delta t_{i}}^{\prime}, \\
\boldsymbol{\Phi}_{\kappa_{\beta, \Delta t_{i}}, x_{t_{0}}} & =\mathbf{H}_{\Delta t_{i}} \boldsymbol{\Phi}_{\kappa_{\beta}, x_{t_{0}}} \text { for } \boldsymbol{\kappa} \beta, \Delta t_{i}=\mathbf{F}_{\Delta t_{i}} \boldsymbol{\kappa}_{\beta}, \\
\mathbf{Q}_{\Delta t_{i}} & =\operatorname{irow}\left[(\mathbf{A} \otimes \mathbf{I}+\mathbf{I} \otimes \mathbf{A})^{-1}\left(\mathbf{A}_{\Delta t_{i}} \otimes \mathbf{A}_{\Delta t_{i}}-\mathbf{I} \otimes \mathbf{I}\right) \operatorname{row}\left(\mathbf{G G}^{\prime}\right)\right] .
\end{aligned}
$$

Equation (5) as such does not differ from the ALT model equation, given by Curran and Bollen (2001). It is only by the restrictions in (6) that the autoregression matrix $\mathbf{A}_{\Delta t_{i}}$ is related to and offers an estimate of the drift matrix $\mathbf{A}$. The same is done by discrete-time exogenous effect matrix $\mathbf{B}_{\Delta t_{i}}$ for the continuous-time exogenous effect matrix $\mathbf{B}$, whereas the discrete-time intercept covariance matrices $\boldsymbol{\Phi}_{\kappa_{\Delta t_{i}}}$ and $\boldsymbol{\Phi}_{\kappa_{\Delta t_{i}}, x_{t_{0}}}$ and slope covariance matrices $\boldsymbol{\Phi}_{\kappa_{\beta, \Delta t_{i}}}, \boldsymbol{\Phi}_{\kappa_{\beta, \Delta t_{i}}, \kappa_{\Delta t_{i}}}$, and $\boldsymbol{\Phi}_{\kappa_{\beta, \Delta t_{i}}, x_{t_{0}}}$ are related to their continuous-time analogues by the intercept constraint matrix $\mathbf{H}_{\Delta t_{i}}$ and slope constraint matrix $\mathbf{F}_{\Delta t_{i}}$, respectively. Finally, (6) specifies how the discrete-time error covariance matrix $\mathbf{Q}_{\Delta t_{i}}$ is related to the diffusion coefficient matrix $\mathbf{G}$. Details can be found in Delsing anf Oud (2008). All expressions in (6) are derived in Oud and Jansen (2000) and Singer (1990); see especially Appendix in the former publication for the derivation of the integrals $\mathbf{B}_{\Delta t_{i}}, \mathbf{H}_{\Delta t_{i}}, \mathbf{F}_{\Delta t_{i}}$, and $\mathbf{Q}_{\Delta t_{i}}$. A detailed explanation how the constrained parameter matrices in (6) are put into SEM matrices to get structural equation modeling (SEM) estimates is found in Oud and Jansen (2000) and Oud (2007). Because the constraints are nonlinear, it is an example of SEM with nonlinear terms as, for example, considered by Klein and Schermelleh-Engel (2010). 


\section{Serious problems of the linear component in the ALT and CALT models}

It is just the presence of the linear components or slope effects $\left(\boldsymbol{\mu}_{\beta}+\boldsymbol{\kappa}_{\beta}\right) t$, with the mean (fixed) slope effects $\boldsymbol{\mu}_{\beta}$ and the random slope variables $\boldsymbol{\kappa}_{\beta}$ containing subject-specific effects that distinguish the ALT and CALT models from standard explanatory models in behavioral science and economics. The random intercept variables $\boldsymbol{\kappa}$ are found elsewhere too (e.g., Hsiao 1986; Oud et al. 1999; Hamerle et al. 1991). The problems of the linear components are not caused by the EDM/SEM procedure, however, which gives correct estimates of the parameters in $\boldsymbol{\mu}_{\beta}$ and in the covariance matrices $\boldsymbol{\Phi}_{\kappa_{\beta}}, \boldsymbol{\Phi}_{\kappa_{\beta}, \kappa}$, and $\boldsymbol{\Phi}_{\kappa_{\beta}, x_{t_{0}}}$. The problems are in the added value of the linear components. Putting the linear components in the same equation as the autoregression matrix or drift matrix makes them competitors of the autoregression and cross-lagged effects (ALT model) or auto- and cross-effects (CALT model) in explaining change over time. In fact, ALT and CALT models are hybrids in that the linear component (LT) competes with the AR component but does not give clear additional information and shows serious disadvantages. Three important specific disadvantages of the linear component in comparison to the AR component will be discussed first. Next, the second-order stochastic differential equation model is presented, which replaces the linear component by the first-order derivative as explanatory variable. The first-order derivative describes the change in the variable it is the derivative of and not more than that and so gives clear new information in comparison to the original variable, which is handled by the AR component. It is shown to lack any of the disadvantages of the linear component.

The first disadvantage of the linear component in comparison to the AR component is that it makes the model unstable by definition. Depending on the eigenvalues, the autoregression or the drift matrix leaves two possibilities: an (asymptotically) stable or unstable model. In general, a stable model is more realistic than an unstable model, as instability leads to explosive behavior (variables going to $+\infty$ or $-\infty$ ), a rare event in practice. So, the AR component makes it possible to check empirically whether the model is stable and all variables converge to a stable equilibrium value. Because of the presence of the linear component, overall means and subject-specific means go to $\infty$ or $-\infty$ necessarily, however, and also the variance necessarily goes to $\infty$. So, the stability check does not make sense, as the state space model (3)-(4) which incorporates a linear component is unstable by definition. As confirmed by Bollen and Curran (2006, pp. 108-109), it does not help to replace the linear component by a higher-order polynomial component, for example, a quadratic or cubic component, or add higher-order components, because all polynomial components make the model unstable by definition. Bollen and Curran (2006, p. 109) confirm that it may be wise to avoid using polynomial components in the modeling of behavioral science processes: "Since most social and psychological processes are bounded, there is an advantage in using a function that does not increase or decrease without limits."

Causal mechanisms can be characterized as "time-specific" or, as it is called in the state-space literature, "nonanticipative." As can be observed in the basic AR feedback process with $x$ influencing $y$ and $y$ influencing $x, y_{t}=a y_{t-1}+b x_{t-1}$ and $x_{t}=c x_{t-1}+d y_{t-1}$, all variables have a time-indication, and there is indeed no anticipation in time. The variables influencing at the right-hand side of the equations are 
located at time point $t-1$, that is, before the value at time point $t$ of the variables to be explained. Reformulating slightly, one gets $y_{t}-y_{t-1}=(a-1) y_{t-1}+b x_{t-1}$ and $x_{t}-x_{t-1}=(c-1) x_{t-1}+d y_{t-1}$. The change variables to be explained at the lefthand side of the equations, $y_{t}-y_{t-1}$ and $x_{t}-x_{t-1}$, come close to slope variables in the LT part of the ALT and CALT models. However, as also observed by Curran and Bollen (2006, p. 208), the latent slope variables $\kappa_{\beta}$ in (1) and (5) differ in one crucial aspect from the change variables. The slope variables characterizing individuals have no time indication and are therefore time-unspecific quantities floating above timespecific reality. It has become customary in the LT literature to compute covariances between the slope variables in $\kappa_{\beta}$, intercept variables in $\kappa$, and between the intercept variables in $\kappa$, on the one hand, and the slope variables in $\kappa_{\beta}$, on the other hand. Often one is specifically interested in the covariances between the intercepts, hypothesized to be causes, and the slopes, hypothesized to be effects. However, the disadvantage of the latent intercept-slope covariances is their time-unspecific character, which makes a causal interpretation impossible and "support" for causal connections unwarranted. In fact, the meaning of the covariances between LT components in the LT as well as ALT and CALT models is unclear.

A third major disadvantage of the linear component is that its sheer presence in the ALT and CALT models makes the models time-varying. Time-invariance in the state-space modeling literature means that the model results are invariant under timescale shifts. This can be clarified again by the basic time-invariant AR equation $y_{t}=$ $a y_{t-1}+b x_{t-1}$ or $y_{t}-y_{t-1}=(a-1) y_{t-1}+b x_{t-1}$. Suppose that intelligence $x_{t-1}$ adds to school achievement, so that the increase in achievement $y_{t}-y_{t-1}$ is equal to the intelligence effect $b x_{t-1}$ plus the auto-effect $(a-1) y_{t-1}$. In a stable model this auto-effect is negative, so that the increase $y_{t}-y_{t-1}$ is positive only if $b x_{t-1}$ is sufficiently positive to exceed the negative $(a-1) y_{t-1}$. Because the model is timeinvariant, we get exactly the same result for difference choices of the zero time point. Suppose that the results for the original time scale $t=\ldots 0,1,2,3 \ldots$ are $\ldots y_{0}=4$, $y_{1}=6, y_{2}=6, y_{3}=8, \ldots$ (on the basis of the parameter values $a=1, b=2$, and exogenous variable values $\left.\ldots x_{0}=1, x_{1}=0, x_{2}=1, \ldots\right)$. If we now shift the time scale by adding 100 to $t$ but keep the model and the intelligence values on the shifted time scale exactly equal, we get exactly equal values $\ldots y_{100}=4, y_{101}=6, y_{102}=6$, $y_{103}=8, \ldots$ on the shifted time scale. Time invariance is generally considered to be a prerequisite for analyzing true causality. If true causality is operating, on the basis of the same intelligence values, the same results should be obtained at different positions on the time scale. But this presupposes that at least the model is time-invariant.

Adding a linear component $\alpha t$ to the basic AR model $y_{t}=a y_{t-1}+b x_{t-1}$, then becoming $y_{t}=a y_{t-1}+b x_{t-1}+\alpha t$, immediately makes the model time-varying, however. If the original results are $\ldots y_{0}=4, y_{1}=9, y_{2}=15, y_{3}=26, \ldots$ for $\alpha=3$ (the rest of the model specified as above), those on the shifted time scale become now $\ldots y_{100}=4, y_{101}=309, y_{102}=615, y_{103}=926, \ldots$, and so are totally different, merely as a result of shifting the time scale. In fact, the time-varying character of the model caused by the linear component implies little empirical for the intercept-slope covariances, computed as part of an LT, ALT, or CALT analysis. These are dependent on the choice of the zero time point in the time scale. By shifting the time scale almost any covariance and correlation value can be reached. For the intercept and slope in the 
same variable, Mehta and West (2000, pp. 25-26) prove for an LT analysis that shifting the time scale so that the original zero time point gets value $\operatorname{cov}\left(\kappa, \kappa_{\beta}\right) / \operatorname{var}\left(\kappa_{\beta}\right)$, where $\boldsymbol{\kappa}$ is the intercept, and $\kappa_{\beta}$ is the slope, makes the new intercept/slope covariance zero. Choosing lower values makes the new intercept/slope covariance positive, and choosing higher value makes it negative. In essence, the proof for intercept and slope in the same variable is given again by Biesanz et al. (2004, p. 50) and by Bollen and Curran (2006, pp. 115-118). As proven in Appendix, however, similar results can be obtained for the intercept in one variable (e.g., intelligence) and slope in a different variable (e.g., achievement). To get a zero intercept/slope covariance or correlation, one replaces the original zero time point by $\operatorname{cov}\left(\kappa_{1}, \kappa_{2 \beta}\right) / \operatorname{cov}\left(\kappa_{1 \beta}, \kappa_{2 \beta}\right)$, where $\kappa_{1}$ is the intercept of one of the variables, and $\kappa_{1 \beta}$ and $\kappa_{2 \beta}$ are the slopes. Choosing lower values makes the new intercept/slope covariance positive again, and choosing higher value makes it negative again, but only if $\operatorname{cov}\left(\kappa_{1 \beta}, \kappa_{2 \beta}\right)$ is positive. If $\operatorname{cov}\left(\kappa_{1 \beta}, \kappa_{2 \beta}\right)$ is negative, covariances take on the opposite signs. We conclude that the height of the covariances is largely an artifact of the choice of the zero time point.

\section{Second-order stochastic differential equation model: replacing the slope variable by the first-order derivative as explanatory variable}

Equations (7)-(8) display the second-order stochastic differential equation model. It is shown how this second-order model, like the CALT model, can be formulated as a special case of the basic continuous-time state space model (3)-(4) and can therefore be estimated by means of the EDM/SEM procedure. Although (7) is specified for two state variables $z_{1}(t)$ and $z_{2}(t)$, it is easily extended for more than two variables or reduced for only one variable. Because in the state vector $\mathbf{x}(t)$ on the right-hand side of (7), in addition to $z_{1}(t)$ and $z_{2}(t)$, the first-order derivatives $\frac{\mathrm{d} z_{1}(t)}{\mathrm{d} t}$ and $\frac{\mathrm{d} z_{2}(t)}{\mathrm{d} t}$ are specified as explanatory variables, in its derivative $\frac{\mathrm{d} \mathbf{x}(t)}{\mathrm{d} t}$ on the lefthand side, the second-order derivatives $\frac{\mathrm{d}^{2} z_{1}(t)}{\mathrm{d} t^{2}}$ and $\frac{\mathrm{d}^{2} z_{2}(t)}{\mathrm{d} t^{2}}$ show up. The 1 's in the matrix A specify that the first-order derivatives on the right-hand side and left-hand side are equal. Because the first-order derivatives are not directly observed, the third and fourth columns in the matrix $\mathbf{C}$ are zero columns.

$$
\begin{aligned}
{\left[\begin{array}{c}
\frac{\mathrm{d} z_{1}(t)}{\mathrm{d} t} \\
\frac{\mathrm{d} z_{2}(t)}{\mathrm{d} t} \\
\frac{\mathrm{d}^{2} z_{1}(t)}{\mathrm{d} t^{2}} \\
\frac{\mathrm{d}^{2} z_{2}(t)}{\mathrm{d} t^{2}}
\end{array}\right] } & =\left[\begin{array}{cccc}
0 & 0 & 1 & 0 \\
0 & 0 & 0 & 1 \\
a_{11} & a_{12} & a_{13} & a_{14} \\
a_{21} & a_{22} & a_{23} & a_{24}
\end{array}\right]\left[\begin{array}{c}
z_{1}(t) \\
z_{2}(t) \\
\frac{\mathrm{d} z_{1}(t)}{\mathrm{d} t} \\
\frac{\mathrm{d} z_{2}(t)}{\mathrm{d} t}
\end{array}\right]+\mathbf{B u}(t)+\mathbf{G} \frac{\mathrm{d} \mathbf{W}(t)}{\mathrm{d} t} \\
\frac{\mathrm{d} \mathbf{x}(t)}{\mathrm{d} t} & = \\
\mathbf{y}_{t_{i}} & =\mathbf{C} \mathbf{x}\left(t_{i}\right)+\mathbf{D u}\left(t_{i}\right)+\mathbf{v}_{t_{i}} .
\end{aligned}
$$




\section{Marital Satisfaction in 235 couples}

The second-order model will be applied to a data set of 235 Dutch couples of husbands and wives, who filled out a questionnaire in four consecutive years (2002, 2003, 2004, 2005). The questionnaire was based on Rusbult (1983) and had Marital Satisfaction as one of the topics. Only the data for Marital Satisfaction (four items that were added into the Marital Satisfaction scale score) of subjects with complete data were analyzed. The matrix $\mathbf{A}$ in the second-order model (7)-(8) is displayed in (9). The model relates the second-order Satisfaction derivative in each partner to her/his own Satisfaction ( $a$ ), to the Satisfaction of her/his partner ( $\alpha a$ or $\beta a$ ), to her/his own first-order Satisfaction derivative $(b)$, and to the first-order Satisfaction derivative of her/his partner $(\alpha b$ or $\beta b$ ). In both partners the effect of the original variable is assumed to be $a$ and of the first-order derivative $b$. As the effect of the partner may be assumed less than one's own effect, $a$ and $b$ are multiplied by a partner effect ( $\alpha$ for the effect of husband on wife, and $\beta$ for the effect of wife on husband).

$$
\begin{gathered}
{\left[\begin{array}{c}
\frac{\mathrm{d} z_{1}(t)}{\mathrm{d} t} \\
\frac{\mathrm{d} z_{2}(t)}{\mathrm{d} t} \\
\frac{\mathrm{d}^{2} z_{1}(t)}{\mathrm{d} t} \\
\frac{\mathrm{d}^{2} z_{2}(t)}{\mathrm{d} t}
\end{array}\right]=\left[\begin{array}{cccc}
0 & 0 & 1 & 0 \\
0 & 0 & 0 & 1 \\
a & \alpha a & b & \alpha b \\
\beta a & a & \beta b & b
\end{array}\right]\left[\begin{array}{c}
z_{1}(t) \\
z_{2}(t) \\
\frac{\mathrm{d} z_{1}(t)}{\mathrm{d} t} \\
\frac{\mathrm{d} z_{2}(t)}{\mathrm{d} t}
\end{array}\right] \begin{array}{c}
a=\text { effect of original variable, } \\
b=\text { effect of first-order deriv- } \\
\text { ative (change variable) } \\
\alpha=\text { effect of husband on wife, } \\
\beta=\text { effect of wife on husband, }
\end{array}} \\
\frac{\mathbf{d} \mathbf{x}(t)}{\mathrm{d} t}= \\
\mathbf{x}(t),
\end{gathered}
$$

The $\chi^{2}$-difference between the $\chi^{2}$ 's of the first-order model and the second-order model is 53.4 , which for $d f=4$, is highly significant. This means that the secondorder model fits significantly better. Quite interesting are the effects between partners $\alpha$ and $\beta$. It turns out that $\alpha$, the effect of husband on wife, is virtually zero, whereas $\beta$, the effect of wife on husband, is -0.384 and significant. Although the latter value is negative, it has to be interpreted in the positive direction, because it multiplies with the negative values $a$ and $b$ into the positive effects $\beta a=0.299$ and $\beta b=2.391$.

Solving the differential equation gives the cross-lagged effects between the original satisfaction variables over increasing intervals but, in a second-order model, also the lagged effect of the first-order derivatives over increasing intervals. Figure 1 displays the lagged effects over increasing intervals of the first-order derivatives (change in Satisfaction in husband and wife) on Satisfaction in wife and husband. Expectedly, the lagged effect of the change in the husband on wife is everywhere negligible. However, there is a lagged effect of Satisfaction change in the wife on husband's Satisfaction, which reaches its maximum after 0.95 year and then, because the model is stable, dies out but quite slowly.

In Fig. 2 the model is used to display in continuous time the mean development in Satisfaction for husbands and wives (original variables) and in Satisfaction change (first-order derivatives). It is shown that during the data collection period (20022005), the mean Satisfaction in wives first rather sharply goes down but then hardly changes any more. The hardly observable decrease is continued in the prediction 


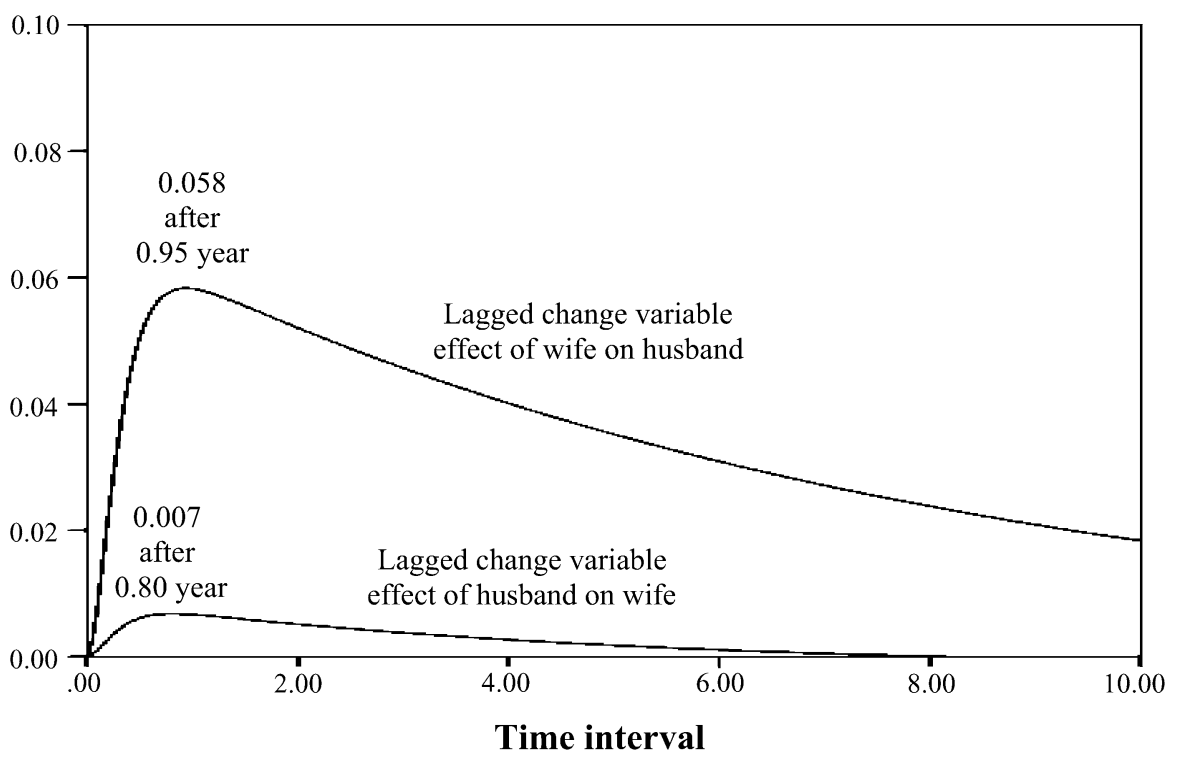

Fig. 1 Lagged change variable effects of husband on wife and wife on husband over increasing intervals; maxima are reached after 0.95 and 0.80 year

period. In husbands, after a very short increase at the start of 2002, a moderate decrease starts which is continued during the prediction period, resulting after several years in a rather big difference in Satisfaction between husbands and wives. Observe that the development in mean Satisfaction for husbands and wives is very precisely reflected in the means of the Satisfaction change variables (first-order derivatives). These are negative over the whole period except at the very start for husbands, indicating that there is almost everywhere decrease in Satisfaction. The very negative first-order derivative in the first months of 2002 for wives corresponds to the sharp decrease in Satisfaction seen there. Very important is that both first-order derivatives converge to 0 , indicating that, in the end, both Satisfaction in wives and in husbands converge to (stable) equilibrium values.

Figure 2 clearly demonstrates that the original variables (top panel in Fig. 2) and the first-order derivatives (bottom panel in Fig. 2) give two different kinds of information about the Satisfaction development, which complement each other. The original variables give information about the level of Satisfaction at each point in time and, multiplied by the coefficients in (9) involving $a(a, \alpha a$, and $\beta a)$, about the level effects. The first-order derivatives give exact information about the Satisfaction change at each point in time and, multiplied by the coefficients in (9) involving $b(b, \alpha b$, and $\beta b$ ), about the change effects. The latter can be different at each point in time, increasing, decreasing, or zero. In contrast, the linear component in CALT model (1) forces the Satisfaction change into the constantly increasing or constantly decreasing linear form, dictated by the constantly increasing time variable $t$.

Whereas the CALT model, because of the linear component in (1), is unstable by definition, the second-order model may be stable or unstable, depending on the data. Because the eigenvalues of the drift matrix in (9) for the Marital Satisfaction data 

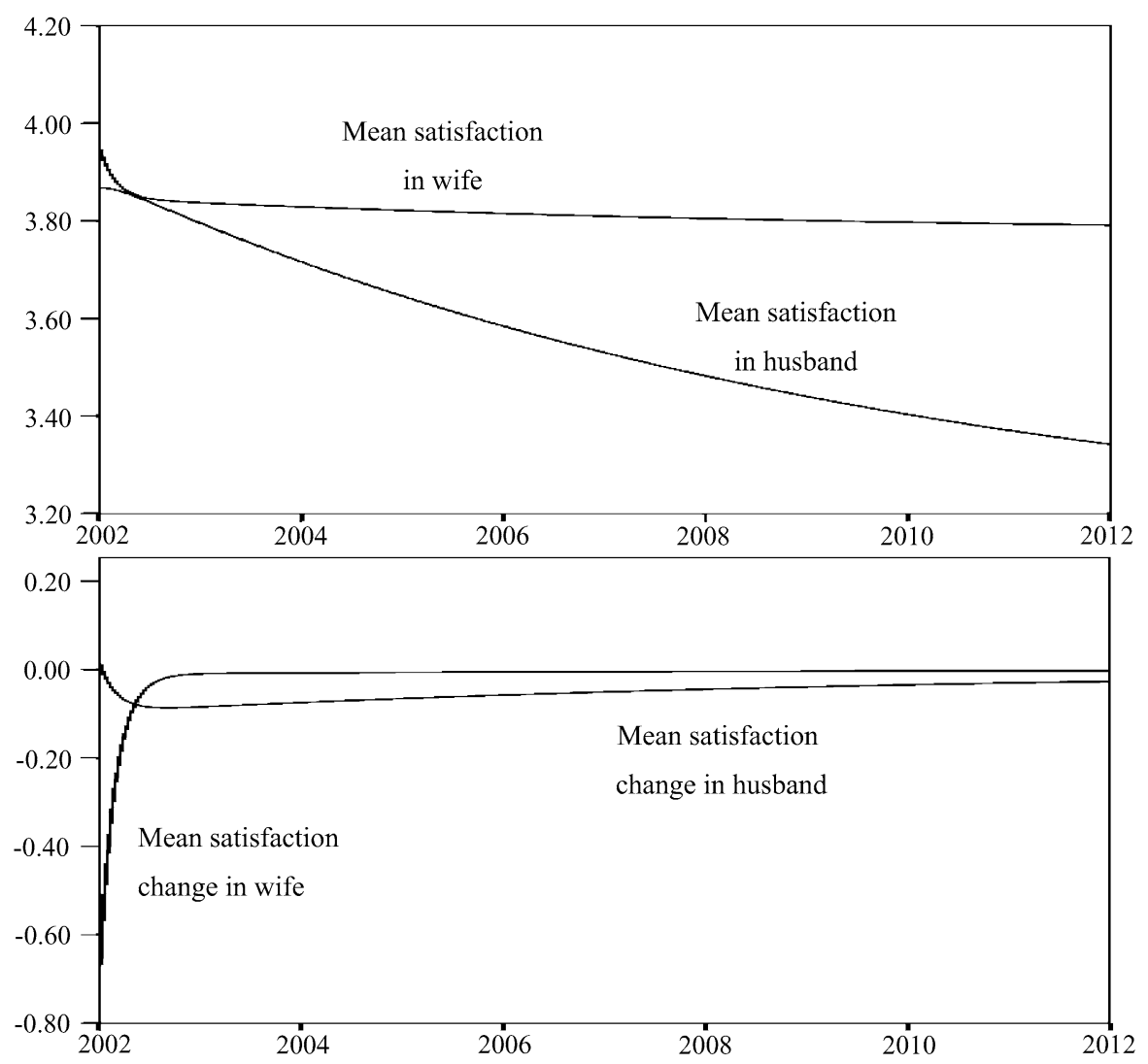

Time

Fig. 2 Model-based estimated, interpolated, and predicted mean development in Satisfaction and Satisfaction change for husbands and wives

have all negative real parts, the model is (asymptotically) stable, and all trajectories converge to stable equilibrium values (e.g., see all curves in Figs. 1 and 2).

Figure 2 clearly demonstrates the time-specific character of the derivatives $\frac{\mathrm{d} z_{1}(t)}{\mathrm{d} t}$ and $\frac{\mathrm{d} z_{2}(t)}{\mathrm{d} t}$ in (9) with different values at different time points (and for different subjects), which is in stark contrast to the time-unspecific (constant) character of the random slope variables $\kappa_{\beta}$ in (1) of the CALT model, which can be different for different subjects but are constant across time.

Finally, because all the coefficients in (9) are constants, none of them a function of time, the second-order model is time-invariant. This means that shifts of the time scale do not affect the model results. Specifically, if for generating Figs. 1 and 2, the model would have used the shifted time scale $02, \ldots, 12$ instead of $2002, \ldots, 2012$, exactly the same figures would have resulted (with time axis 2002, ., 2012 replaced by $02, \ldots, 12)$. 


\section{Discussion}

After introducing the CALT model, which is a continuous-time version of the ALT model, this paper sharply criticized the linear component in the ALT and CALT models. The models are hybrids, with the linear component competing with the autoregressive component and not giving clear additional information. Further, the linear component (1) makes the model unstable by definition, (2) by its time-unspecific character, it is unsuitable for assessing causal relationships, (3) it makes the model time-varying and relationships dependent on the choice of the zero time point. The introduction of the second-order stochastic differential equation model as an alternative for the ALT and CALT models was followed by an example, using the first-order derivative as an explanatory variable instead of the linear component. The first-order derivative (1) leaves the possibility of a stable or unstable model open, (2) is timespecific as it takes on different values across time, and (3) its effects are not affected by the choice of different zero time points.

Finally, it should be noted that the criticisms (1), (2), and (3) do not apply only to the linear components in the ALT and CALT models but more generally to the linear and higher-order polynomial components in the latent trajectory model (LT) as well. However, the emphasis on the CALT model is motivated by the alternative of the AR component offered by this model in the same equation and by the possibility to transform it into the second-order model by replacing the linear component by the first-order derivative. The CALT model might also first be fitted to the data, before trying to improve the fit by the second-order model.

Open Access This article is distributed under the terms of the Creative Commons Attribution Noncommercial License which permits any noncommercial use, distribution, and reproduction in any medium, provided the original author(s) and source are credited.

\section{Appendix: How to shift the zero time point in the LT model to get a zero intercept/slope covariance in the same variable (intercept and slope of $x_{1}$ ) or between different variables (intercept of $x_{1}$ and slope of $x_{2}$ )}

The procedure will be explained for a model in five time points with equal intervals $(t=0,1,2,3,4)$ to be shifted toward $t^{\prime}=0+a, 1+a, 2+a, 3+a, 4+a$. However, the restriction to less than or extension to more than five time points and handling of unequal intervals (e.g., $t=0,2,3,6,10$ ) are straightforward. A linear LT model consists of three matrices, loading matrix $\Lambda$, latent covariance matrix $\boldsymbol{\Psi}$, and measurement error covariance matrix $\boldsymbol{\Theta}$, that combine into the model-implied moment matrix

$$
\Sigma=\Lambda \Psi \Lambda^{\prime}+\Theta
$$

Only the matrices

$$
\boldsymbol{\Lambda}=\left[\begin{array}{llll}
1 & 0 & 1 & 0 \\
1 & 1 & 1 & 1 \\
1 & 2 & 1 & 2 \\
1 & 3 & 1 & 3 \\
1 & 4 & 1 & 4
\end{array}\right]
$$


and

$$
\boldsymbol{\Psi}=\left[\begin{array}{cccc}
\operatorname{var}\left(\kappa_{1}\right) & & & \\
\operatorname{cov}\left(\kappa_{1}, \kappa_{1 \beta}\right) & \operatorname{var}\left(\kappa_{1 \beta}\right) & & \\
\operatorname{cov}\left(\kappa_{1}, \kappa_{2}\right) & \operatorname{cov}\left(\kappa_{2}, \kappa_{1 \beta}\right) & \operatorname{var}\left(\kappa_{2}\right) & \\
\operatorname{cov}\left(\kappa_{1}, \kappa_{2 \beta}\right) & \operatorname{cov}\left(\kappa_{1 \beta}, \kappa_{2 \beta}\right) & \operatorname{cov}\left(\kappa_{2}, \kappa_{2 \beta}\right) & \operatorname{var}\left(\kappa_{2 \beta}\right)
\end{array}\right]
$$

are involved in the procedure, however. Transformation from $\boldsymbol{\Lambda}$ to $\boldsymbol{\Lambda}^{*}=\boldsymbol{\Lambda} \mathbf{T}$,

$$
\boldsymbol{\Lambda}^{*}=\left[\begin{array}{llll}
1 & 0+a & 1 & 0+a \\
1 & 1+a & 1 & 1+a \\
1 & 2+a & 1 & 2+a \\
1 & 3+a & 1 & 3+a \\
1 & 4+a & 1 & 4+a
\end{array}\right]
$$

is done by transformation matrix $\mathbf{T}$,

$$
\mathbf{T}=\left[\begin{array}{llll}
1 & a & 0 & 0 \\
0 & 1 & 0 & 0 \\
0 & 0 & 1 & a \\
0 & 0 & 0 & 1
\end{array}\right] \rightarrow \mathbf{T}^{-1}=\left[\begin{array}{cccc}
1 & -a & 0 & 0 \\
0 & 1 & 0 & 0 \\
0 & 0 & 1 & -a \\
0 & 0 & 0 & 1
\end{array}\right]
$$

To keep the same $\boldsymbol{\Sigma}$,

$$
\boldsymbol{\Sigma}=\boldsymbol{\Lambda} \boldsymbol{\Psi} \boldsymbol{\Lambda}^{\prime}+\boldsymbol{\Theta}=\boldsymbol{\Lambda}^{*} \boldsymbol{\Psi}^{*} \boldsymbol{\Lambda}^{* \prime}+\boldsymbol{\Theta},
$$

$\boldsymbol{\Psi}$ needs to be transformed too:

$$
\boldsymbol{\Psi}^{*}=\mathbf{T}^{-1} \boldsymbol{\Psi} \mathbf{T}^{-1 \prime}
$$

Therefore,

$$
\begin{aligned}
& \operatorname{cov}^{*}\left(\kappa_{1}, \kappa_{1 \beta}\right)=\operatorname{cov}\left(\kappa_{1}, \kappa_{1 \beta}\right)-a \operatorname{var}\left(\kappa_{1 \beta}\right)=0 \rightarrow a=\frac{\operatorname{cov}\left(\kappa_{1}, \kappa_{1 \beta}\right)}{\operatorname{var}\left(\kappa_{1 \beta}\right)} \\
& \operatorname{cov}^{*}\left(\kappa_{1}, \kappa_{2 \beta}\right)=\operatorname{cov}\left(\kappa_{1}, \kappa_{2}\right)-a \operatorname{cov}\left(\kappa_{1 \beta}, \kappa_{2 \beta}\right)=0 \rightarrow a=\frac{\operatorname{cov}\left(\kappa_{1}, \kappa_{2 \beta}\right)}{\operatorname{cov}\left(\kappa_{1 \beta}, \kappa_{2}\right)} .
\end{aligned}
$$

This proves that shifting $t=0,1,2,3,4$ to $t^{\prime}=0+a, 1+a, 2+a, 3+a, 4+a$ with choice $a=\frac{\operatorname{cov}\left(\kappa_{1}, \kappa_{1 \beta}\right)}{\operatorname{var}\left(\kappa_{1 \beta}\right)}$ makes the covariance/correlation between intercept $\kappa$ and slope $\kappa_{\beta}$ in the same variable zero, while the choice $\left.a=\frac{\operatorname{cov}\left(\kappa_{1}, \kappa_{2 \beta}\right)}{\operatorname{cov}\left(\kappa_{1 \beta}, \kappa_{2} \beta\right.}\right)$ makes the covariance between intercept $\kappa$ in one variable and slope $\kappa_{\beta}$ in the other variable zero.

\section{References}

Arnold, L.: Stochastic Differential Equations. Wiley, New York (1974)

Bergstrom, A.R.: The history of continuous-time econometric models. Econom. Theory 4, 365-383 (1988) 
Biesanz, J.C., Deeb-Sossa, N., Papadakis, A.A., Bollen, K.A., Curran, P.J.: The role of coding time in estimating and interpreting growth curve models. Psychol. Methods 9, 30-52 (2004)

Bollen, K.A., Curran, P.J.: Autoregressive latent trajectory (ALT) models: a synthesis of two traditions. Sociol. Methods Res. 32, 336-383 (2004)

Bollen, K.A., Curran, P.J.: Latent Curve Models: A Structural Equation Perspective. Wiley, Hoboken (2006)

Curran, P., Bollen, K.: The best of both worlds: combining autoregressive and latent curve models. In: Sayer, A., Collins, L. (eds.): New Methods for the Analysis of Change, pp. 107-135. American Psychological Association, Washington (2001)

Delsing, M.J.M.H., Oud, J.H.L.: Continuous time modeling of panel data: SEM versus filter techniques. Stat. Neerl. 62, 58-82 (2008)

Hamerle, A., Nagl, W., Singer, H.: Problems with the estimation of stochastic differential equations using structural equation models. J. Math. Soc. 16, 201-220 (1991)

Hsiao, C.: Analysis of Panel Data. Cambridge University Press, Cambridge (1986)

Klein, A.G., Schermelleh-Engel, K.: Introduction of a new measure for detecting poor fit due to omitted nonlinear terms in SEM. Adv. Stat. Anal. (2010, this issue)

Kuo, H.-H.: Introduction to Stochastic Integration. Springer, New York (2006)

Mehta, P.D., West, S.G.: Putting the individual back in individual growth curves. Psychol. Methods 5, 23-43 (2000)

Oud, J.H.L.: Continuous time modeling of reciprocal relationships in the cross-lagged panel design. In: Boker, S.M., Wenger, M.J. (eds.): Data Analytic Techniques for Dynamical Systems, pp. 87-129. Lawrence Erlbaum Associates, Mahwah (2007)

Oud, J.H.L., Jansen, R.A.R.G.: Continuous time state space modeling of panel data by means of SEM. Psychometrika 65, 199-215 (2000)

Oud, J.H.L., Jansen, R.A.R.G., van Leeuwe, J.F.J., Aarnoutse, C.A.J., Voeten, M.J.M.: Monitoring pupil development by means of the Kalman filter and smoother based upon SEM state space modeling. Learn. Individ. Differ. 11, 121-136 (1999)

Rusbult, C.E.: A longitudinal test of the investment model: the development (and deterioration) of satisfaction and commitment in heterosexual involvements. J. Pers. Soc. Psychol. 4 5, 101-117 (1983)

Ruymgaart, P.A., Soong, T.T.: Mathematics of Kalman-Bucy Filtering. Springer, Berlin (1985)

Singer, H.: Parameterschätzung in Zeitkontinuierlichen Dynamischen Systemen [Parameter Estimation in Continuous-Time Dynamic Systems]. Hartung-Gorre, Konstanz (1990) 